\title{
UNIMODULAR MATRICES IN BANACH ALGEBRA THEORY
}

\author{
GUSTAVO CORACH AND ANGEL R. LAROTONDA
}

\begin{abstract}
Let $A$ be a ring with 1 and denote by $L$ (resp. $R$ ) the set of left (resp. right) invertible elements of $A$. If $A$ has an involution *, there is a natural bijection between $L$ and $R$. In general, it seems that there is no such bijection; if $A$ is a Banach algebra, $L$ and $R$ are open subsets of $A$, and they have the same cardinality. More generally, we prove that the spaces $U_{k}\left(A^{n}\right)$ of $n \times k$-left-invertible matrices and ${ }_{k} U\left(A^{n}\right)$ of $k \times n$-right-invertible matrices are homotopically equivalent. As a corollary, we answer negatively two questions of Rieffel [12].
\end{abstract}

1. Let $A$ be a ring (with an identity element 1 ) and $k \leqslant n$. A matrix $x \in A^{n \times k}$ is unimodular if there exists $y \in A^{k \times n}$ such that $y \cdot x=1 \in M_{k}(A)=A^{k \times k}$. We denote by $U_{k}\left(A^{n}\right)$ the set of all unimodular matrices of $A^{n \times k}$, and $S_{k}\left(A^{n}\right)=\{(x, y)$ $\left.\in A^{n \times k} \times A^{k \times n}: y \cdot x=1\right\}$. The first projection induces a mapping $p: S_{k}\left(A^{n}\right) \rightarrow$ $U_{k}\left(A^{n}\right)$.

1.1. Proposition. Let $A$ be a ring, $x \in A^{n \times k}$, and define $T_{x}: M_{n}(A) \rightarrow A^{n \times k}$ by $T_{x}(\sigma)=\sigma x$. Then $x$ is unimodular if and only if $T_{x}$ is a direct epimorphism; i.e., there is an A-linear map $S: A^{n \times k} \rightarrow M_{n}(A)$ such that $T_{x} \circ S=1_{A^{n \times k}}$.

Proof. Obvious.

From now on, $A$ denotes a (real or complex) Banach algebra. Our purpose is to study the fibration properties of the mappings

$$
t_{x}=T_{x} \mid \mathrm{GL}_{n}(A): \mathrm{GL}_{n}(A) \rightarrow U_{k}\left(A^{n}\right),
$$

$$
\text { for } x \in U_{k}\left(A^{n}\right) \text { and } p: S_{k}\left(A^{n}\right) \rightarrow U_{k}\left(A^{n}\right) \text {. }
$$

1.2. Lemma. (i) $U_{k}\left(A^{n}\right)$ is open in $A^{n \times k}$.

(ii) $t_{x}$ is continuous, for every $x \in U_{k}\left(A^{n}\right)$.

(iii) $p$ is continuous.

Proof. It is clear that $t_{x}$ and $p$ are continuous, because they are restrictions of linear mappings. If $x_{0} \in A^{n \times k}$ is unimodular and $y \cdot x_{0}=1 \in M_{k}(A)$, there is a neighborhood $U$ of $x_{0}$ in $A^{n \times k}$ such that $y \cdot x \in \mathrm{GL}_{k}(A)$ for every $x \in U$, for the set of invertible elements of a Banach algebra is open. But it is evident that $U$ is contained in $U_{k}\left(A^{n}\right)$. Thus $U_{k}\left(A^{n}\right)$ is open as claimed.

In view of the theory of Banach manifolds, as developed in Lang [7], we can prove the next result. Recall that a differentiable map $f: X \rightarrow Y$ is a submersion at $x \in X$ if the derivative of $f$ at $x \in X$ is a direct epimorphism from the tangent space of $X$ at $x$ onto the tangent space of $Y$ at $f(x)$.

Received by the editors June 19, 1984 and, in revised form, March 1, 1985.

1980 Mathematics Subject Classification. Primary 46H05, 18F25; Secondary 55R10, 55P10, 16A25. 
1.3. Proposition. Let $A$ be a Banach algebra and $x \in A^{n \times k}$. Then the following hold.

(1) If $x \in U_{k}\left(A^{n}\right)$, then $t_{x}: \mathrm{GL}_{n}(A) \rightarrow U_{k}\left(A^{n}\right)$ is a submersion.

(2) $S_{k}\left(A^{n}\right)$ is a Banach manifold.

Proof. (1) If $x \in U_{k}\left(A^{n}\right)$, the derivative of $t_{x}$ at any $\sigma \in \mathrm{GL}_{n}(A)$ coincides with $T_{x}$, which is a direct epimorphism, by 1.1 , so $t_{x}$ is a submersion at $\sigma$.

(2) Following Raeburn [11], we need only prove that the derivative of $\phi$ : $A^{n \times k} \times A^{k \times n} \rightarrow M_{k}(A)$, defined by $\phi(x, y)=y \cdot x$, at any $\left(x_{0}, y_{0}\right) \in S_{k}\left(A^{n}\right)$ is a direct epimorphism for $S_{k}\left(A^{n}\right)=\phi^{-1}(1)$.

Now, the derivative of $\phi$ at $\left(x_{0}, y_{0}\right)$ is the $A$-linear map $(w, z) \rightarrow z \cdot x_{0}+y_{0} \cdot w$ $\left(w \in A^{n \times k}, z \in Z^{k \times n}\right)$, and the map $a \rightarrow 1 / 2\left(x_{0} a, a y_{0}\right)\left(a \in M_{k}(A)\right)$ is a right inverse of $D \phi\left(x_{0}, y_{0}\right)$.

1.4. Corollary. For every $x \in U_{k}\left(A^{n}\right)$, the map $t_{x}: \mathrm{GL}_{n}(A) \rightarrow U_{k}\left(A^{n}\right)$ is open, and its image $\mathrm{GL}_{n}(A) \cdot x$ is open and closed in $U_{k}\left(A^{n}\right)$.

Proof. Clearly a submersion is an open map. Thus, for every $z \in U_{k}\left(A^{n}\right)$, $\mathrm{GL}_{n}(A) \cdot z$ is open in $U_{k}\left(A^{n}\right)$. But $\mathrm{GL}_{n}(A) \cdot z$ is the orbit of $z$ under the action of $\mathrm{GL}_{n}(A)$ on $U_{k}\left(A^{n}\right)$ given by left multiplication, so it is closed in $U_{k}\left(A^{n}\right)$ : in fact, when all orbits are open, they are also closed.

1.5. Remarks. (1) Observe that, if $z \in U_{k}\left(A^{n}\right)$ belongs to the orbit $\mathrm{GL}_{n}(A) \cdot x$, the entire connected component of $z$ in $U_{k}\left(A^{n}\right)$ is contained in the same orbit. This obvious (in view of 1.4) fact has interesting consequences, as we shall see.

(2) If in the proof of 1.4 we consider the action of $\mathrm{GL}_{n}(A)_{0}$, the connected component of the neutral element, on $U_{k}\left(A^{n}\right)$, we get that the connected component of $x$ in $U_{k}\left(A^{n}\right)$ is $\mathrm{GL}_{n}(A)_{0} \cdot x$.

1.6. TheOREM. Let $A$ be a Banach algebra and $x \in U_{k}\left(A^{n}\right)$. Then the following hold.

(i) $t_{x}$ induces a principal locally trivial fibre bundle $\mathrm{GL}_{n}(A) \rightarrow \mathrm{GL}_{n}(A) \cdot x$.

(ii) $t_{x}: \mathrm{GL}_{n}(A) \rightarrow U_{k}\left(A^{n}\right)$ is a Serre fibration.

(iii) $t_{x}$ is surjective if and only if the induced mapping $\pi_{0}\left(t_{x}\right): \pi_{0}\left(\mathrm{GL}_{n}(A)\right) \rightarrow$ $\pi_{0}\left(U_{k}\left(A^{n}\right)\right)$ is onto. In particular, $t_{x}$ is surjective if $U_{k}\left(A^{n}\right)$ is connected.

Proof. Let $G_{x}$ be the stabilizer of $x$; i.e., $G_{x}=\left\{\sigma \in \mathrm{GL}_{n}(A): \sigma \cdot x=x\right\}$. Then $G_{x}$ is a Banach-Lie subgroup of $\mathrm{GL}_{n}(A)$, and, by $1.4, \mathrm{GL}_{n}(A) \cdot x$ may be identified with the homogeneous space $\mathrm{GL}_{n}(A) / G_{x}$. So, assertion (i) follows from the general theory of Banach-Lie groups as Bourbaki [1] or Raeburn [11].

For the proof of (ii), it is well known that any locally trivial bundle over a paracompact base space is a Serre fibration (see Dold [3]). In our case, $U_{k}\left(A^{n}\right)$ is metrizable, a fortiori paracompact. The proof of (iii) is now obvious.

The problem of determining whether an element $z$ of $U_{k}\left(A^{n}\right)$ belongs to $\mathrm{GL}_{n}\left(A^{n}\right)$ - $x$ for a fixed $x \in U_{k}\left(A^{n}\right)$ is, in general, very difficult. Let us give an example. Let $A$ be the algebra of real continuous functions on the unit sphere $S^{n-1}$ of $R^{n}$, and let $x_{i}, i=1, \ldots, n$, be the coordinate functions. Clearly, $x=\left(x_{1}, \ldots, x_{n}\right)$ belongs to 
$U_{1}\left(A^{n}\right)$ for $x \cdot{ }^{t} x=\sum_{i=1}^{n} x_{i}^{2}=1$. Let $t$ be the map $\sigma \rightarrow \sigma \cdot e_{1}$ (where $e_{1}$ is the first canonical vector of $A^{n}$ ) from $\mathrm{GL}_{n}(A)$ into $U_{1}\left(A^{n}\right)$. We shall show that $x$ belongs to $\mathrm{GL}_{n}(A) \cdot e_{1}$ only if $n=2,4$, or 8 . In fact, $x \in \mathrm{GL}_{n}(A) \cdot e_{1}$ means that there is a basis $\left\{v_{1}, v_{2}, \ldots, v_{n}\right\}$ of $A^{n}$ with $v_{1}=x$. Let $S$ be the free supplement of $A \cdot x$ generated by $\left\{v_{2}, \ldots, v_{n}\right\}$ and $S^{\prime}=\left\{v \in A^{n}:\langle v, x\rangle=0\right\}$. Then $S^{\prime}$ is another supplement of $A \cdot x$, so $S$ and $S^{\prime}$ are isomorphic and $S^{\prime}$ must be free. But $S^{\prime}$ identifies with the $A$-module of continuous sections of the tangent bundle $\tau\left(S^{n-1}\right)$ of $S^{n-1}$ (see Swan [13]). Thus, since $S^{\prime}$ is free, $\tau\left(S^{n-1}\right)$ must be a trivial bundle, and this may happen only if $n=2,4$, or 8 , as claimed.

Let $A$ be a ring, and let $e$ be the $n \times k$-matrix whose columns are, in order, the first $k$ canonical vectors of $A^{n}$. Let us set $t=t_{e}: \mathrm{GL}_{n}(A) \rightarrow U_{k}\left(A^{n}\right) . A$ is $(n, k)$-Hermite if $t$ is onto. (This terminology is consistent with that of Lam [6], at least for commutative rings.) The following lemma shows that the choice of $e$ is irrelevant.

1.7. Lemma. Let $A$ be a ring. Then $A$ is $(n, k)$-Hermite if and only if $t_{x}$ : $\mathrm{GL}_{n}(A) \rightarrow U_{k}\left(A^{n}\right)$ is onto for every $x \in U_{k}\left(A^{n}\right)$.

Proof. Suppose that $A$ is $(n, k)$-Hermite, and take $z$ and $x$ in $U_{k}\left(A^{n}\right)$. Then there exist $\tau$ and $\sigma$ in $\mathrm{GL}_{n}(A)$ such that $z=\tau \cdot e$ and $x=\sigma \cdot e$; then $t_{x}\left(\tau \sigma^{-1}\right)=\tau \cdot \sigma^{-1}$ $\cdot x=z$. The converse is trivial.

From 1.6(iii) we get

1.8. Proposition. Let $A$ be a Banach algebra. Then $A$ is $(n, k)$-Hermite if and only if $\pi_{0}(t)$ is onto. In particular, $A$ is $(n, k)$-Hermite if $U_{k}\left(A^{n}\right)$ is connected.

1.9. Examples. (1) Let $A$ be a complex commutative Banach algebra with spectrum $X(A)$. It is well known that $\pi_{0}\left(U_{k}\left(A^{n}\right)\right)$ coincides with the set of homotopy classes of maps from $X(A)$ into $U_{k}\left(\mathbf{C}^{n}\right)$ (see Novodvorski [9], Taylor [14] and Raeburn [11]). Lin [8] proved that $A$ is $(n, k)$-Hermite if and only if $C(X(A))$, the algebra of complex continuous functions on $X(A)$, is; moreover, for $x$ and $z$ in $U_{k}\left(A^{n}\right), z$ belongs to $\mathrm{GL}_{n}(A) \cdot x$ if and only if the Gelfand transform $\hat{z}$ belongs to $\mathrm{GL}_{n}(C(X(A))) \cdot \hat{x}$. In particular, if $X(A)$ is dominated by an $m$-dimensional space, with $m \leqslant 2(n-k)$, then $A$ is $(n, k)$-Hermite. This last fact has also been proved by Lin [8].

(2) Let $\mathscr{H}$ be a Hilbert space and $A$ the algebra of bounded linear operators on $\mathscr{H}$. Then $\mathrm{GL}_{n}(A)$ is contractible for every $n$ (Kuiper [5]). Now, $A$ is isomorphic to $A^{n \times k}$ (as $A$-modules and as Banach spaces), and it is easy to see that $U_{k}\left(A^{n}\right)$ is homeomorphic to $U_{1}\left(A^{1}\right)$, which is disconnected, as we shall see 2.6(iii). Then $A$ cannot be $(n, k)$-Hermite for any $k \leqslant n$.

(3) If the stable range of $A$ is $\leqslant n-k, A$ is $(n, k)$-Hermite (see Corach and Larotonda [21]).

2. In this section we study the fibration properties of the map $p: S_{k}\left(A^{n}\right) \rightarrow U_{k}\left(A^{n}\right)$ when $A$ is a Banach algebra. 
2.1. TheOREM. Let $A$ be a Banach algebra. Then the projection onto the first coordinate $p: S_{k}\left(A^{n}\right) \rightarrow U_{k}\left(A^{n}\right)$ is a locally trivial bundle with contractible fibre.

Proof. For $\left(x_{0}, y_{0}\right) \in S_{k}\left(A^{n}\right)$ we define $\phi: A^{n \times k} \rightarrow \mathrm{GL}_{n}(A) \cdot x_{0} \subset A^{n \times k}$ by $\phi(z)=\exp \left(x, y_{0}\right) \cdot x_{0}$. The derivative of $\phi$ at $0 \in A^{n \times k}$ is the identity map of $A^{n \times k}$, so by the inverse function theorem $[1,7]$ there exist neighborhoods $U$ of 0 in $A^{n \times k}$ and $V$ of $x_{0}$ in $U_{k}\left(A^{n}\right)$ such that $\phi$ is a diffeomorphism from $U$ onto $V$. We define a section of $t_{x_{0}}$ over $V, s: V \rightarrow \mathrm{GL}_{n}(A)$, by $s(x)=\exp \left(\phi^{-1}(x) \cdot y_{0}\right):$ In fact, $t_{x} \circ s(x)$ $=s(x) \cdot x_{0}=x$ for every $x \in V$ and $s\left(x_{0}\right)=1_{M_{n}(A)}$.

We now define a trivializing map $g: p^{-1}(V) \rightarrow V \times N$ by $g(x, y)=(x, y \cdot s(x)$ $\left.-y_{0}\right)$, where $N=\left\{y \in A^{k \times n}: y \cdot x_{0}=0\right\}$; the inverse of $g$ is $h: V \times N \rightarrow p^{-1}(V)$, $h(x, z)=\left(x,\left(z+y_{0}\right) \cdot s(x)^{-1}\right)$. The fibre $p^{-1}(x)$, for $x \in V$, is the affine manifold $\left\{\left(x,\left(y+y_{0}\right) \cdot s(x)^{-1}\right): y \in N\right\}$, obviously contractible.

2.2. Corollary. The projection $p$ is a homotopy equivalence and it admits a global section.

Proof. From the examination of the homotopy sequence of $p$, it follows that $p$ is a weak homotopy equivalence, so, by a result of Palais [10, Theorem 15], since $S_{k}\left(A^{n}\right)$ and $U_{k}\left(A^{n}\right)$ are metrizable manifolds (by 1.2 and 1.3), $p$ is a homotopy equivalence. The second assertion follows from a result of Godement (see Dold [3, p. 223]) which states that a fibre bundle with contractible fibre over a paracompact space has a global section.

2.3. Corollary. For every compact pair $(Z, Y)$, every map $f:(Z, Y) \rightarrow$ $\left(U_{k}\left(A^{n}\right), x_{0}\right)$, and every $y_{0} \in A^{k \times n}$ such that $y_{0} \cdot x_{0}=1 \in M_{k}(A)$, there is a lifting $\bar{f}$ : $(Z, Y) \rightarrow\left(S_{k}\left(A^{n}\right),\left(x_{0}, y_{0}\right)\right)$; i.e., $p \bar{f}=f$.

2.4. Corollary. For every compact space $X$ the natural inclusion $U_{k}\left(A(X)^{n}\right) \rightarrow$ $C\left(X, U_{k}\left(A^{n}\right)\right.$ ) (where $A(X)$ is the algebra of maps from $X$ into $\left.A\right)$ is a homeomorphism.

We have been studying left unimodular matrices. We can also consider right unimodular matrices: $y \in A^{k \times n}(k \leqslant n)$ is right unimodular if there exists $x \in A^{n \times k}$ such that $y \cdot x=1 \in M_{k}(A)$. Of course, the set ${ }_{k} U\left(A^{n}\right)$ of right unimodular matrices is the image of the second projection $p_{2}: S_{k}\left(A^{n}\right) \rightarrow A^{k \times n}$. We may reproduce all the results obtained for $U_{k}\left(A^{n}\right)$ in this new context. In particular, we may prove that $p_{2}: S_{k}\left(A^{n}\right) \rightarrow{ }_{k} U\left(A^{n}\right)$ is a homotopy equivalence. Thus, we have

2.5. Corollary. $U_{k}\left(A^{n}\right)$ and ${ }_{k} U\left(A^{n}\right)$ are homotopically equivalent.

REMARK. This result answers negatively question 4.8 of Rieffel [12] and generalizes his Proposition 10.2 to any Banach algebra $A$.

We now consider the case $n=k=1$, so $U_{1}\left(A^{1}\right)=L$ and ${ }_{1} U\left(A^{1}\right)=R$.

2.6. TheOREM. Let $A$ be a Banach algebra. Then the following hold.

(i) $L$ and $R$ are homotopically equivalent.

(ii) The connected components of 1 in $L, R$ and $G$ (= group of units of $A$ ) coincide; in particular, $G$ is connected if $L$ or $R$ are connected and, in this case, $G=L=R$. Thus, $L$ and $R$ are disconnected if $G \neq L$ or $G \neq R$. 
The proof follows directly from 2.5 and the following obvious lemma.

2.7. LeMMA. $G$ is open and closed in $L$ and $R$.

\section{REFERENCES}

1. N. Bourbaki, Varietés, Fascicule de résultats, Hermann, Paris, 1967.

2. G. Corach and A. R. Larotonda, A stabilization theorem for Banach algebras, J. Algebra (to appear).

3. A. Dold, Partitions of unity in the theory of fibrations, Ann. of Math. (2) 78 (1963), 223-255.

4. S. T. Hu, Homotopy theory, Academic Press, New York, 1959.

5. N. Kuiper, The homotopy type of the unitary group of Hilbert space, Topology 3 (1965), 19-30.

6. T. Y. Lam, Serre's conjecture, Lecture Notes in Math., vol. 635, Springer-Verlag, Berlin and New York, 1978.

7. S. Lang, Differentiable manifolds, Addison-Wesley, Reading, Mass., 1972.

8. V. Ya. Lin, Holomorphic fibering and multivalued functions of elements of a Banach algebra, Funct. Anal. Appl. 7 (1973), 122-128.

9. M. E. Novodvorski, Certain homotopical invariants of spaces of maximal ideals, Mat. Zametki (1967), 487-494.

10. R. S. Palais, Homotopy theory of infinite dimensional manifolds, Topology 5 (1966), 1-16.

11. I. Raeburn, The relationship between a commutative Banach algebra and its maximal ideal space, $\mathrm{J}$. Funct. Anal. 25 (1978), 366-390.

12. M. Rieffel, Dimension and stable rank in the K-theory of $C^{*}$-algebras, Proc. London Math. Soc. (3) 46 (1983), 301-333.

13. R. Swam, Vector bundles and projective bundles, Trans. Amer. Math. Soc. 105 (1962), 264-277.

14. J. L. Taylor, Topological invariants of the maximal ideal space of a Banach algebra, Adv. in Math. 19 (1976), 149-206.

Instituto Argentino de Matemática, Viamonte 1636, 1055 Buenos Aires, Argentina

Departamento de Matemátics, facultad de Ciencias Exactas, Universidad de Buenos Aires, Ciudad Universitaria, 1428 Buenos Aires, Argentina 